\title{
Stability, dynamics of convergence and tuning of observer-based kinetics estimators
}

\author{
R. Oliveira ${ }^{\text {a }}$ E.C. Ferreira ${ }^{\mathrm{b}}$, S. Feyo de Azevedo ${ }^{\mathrm{a}, *}$ \\ ${ }^{a}$ Department of Chemical Engineering, Faculty of Engineering, University of Porto, Rua Dr. Roberto Frias, 4200-465 Porto, Portugal \\ ${ }^{\mathrm{b}}$ Centro de Engenharia Biológica- IBQF, Universidade do Minho, 4710-057 Braga, Portugal
}

\begin{abstract}
This work discusses issues concerning stability, tuning and dynamics of convergence of observer-based kinetics estimators. The analysis focuses on both continuous and discrete time formulations of the estimation algorithms. Concerning the former, it is shown that, with proper tuning, stability can be guaranteed, while simultaneously imposing a desired quasi-time invariant second order time response for the convergence of estimates to true values. Concerning the latter, an algorithm is presented, based on a forward Euler discretisation, whose error system is shown to be linear time-invariant. Furthermore, stability conditions were derived, which define the stable domain for the discretisation period as function of the tuning parameters. The theory is illustrated with a case-study of Baker's yeast fermentation. Results clearly confirm the theoretical developments. In particular, results concerning the stability domain for the Euler-based discrete formulation of the estimator are shown to have relevant practical implications. (C) 2002 Elsevier Science Ltd. All rights reserved.
\end{abstract}

Keywords: Observer-based estimator; Stability; Dynamics of convergence; Tuning; Baker's yeast

\section{Introduction}

The development of bioprocess monitoring and control strategies relying on mathematical (phenomenological) description of micro-organism growth kinetics is a critical issue in bioprocess model-based operation. Mainly because mechanisms for cell growth and product formation are rather complex, kinetic models for bioprocess design, monitoring and control assume, traditionally, simplified unstructured and non-segregated descriptions of the cell system. Unfortunately, in many cases, such models are not accurate enough for solving the problems in study. The other critical issue is related to the identification of kinetic parameters. Even for those simplified models, parameter identification requires a careful and expensive experimental planning [1-3]. For these two reasons, quite frequently the benefit/cost ratio associated to such developments hinders their application in the biochemical industries. In some cases it may be possible to employ, at reasonable developing costs, algorithms for accurate kinetic/parameter estimation that rely on on-line process measurements and that do not assume the knowledge of the underlying kinetic

\footnotetext{
* Corresponding author. Fax: + 351-22-508-1632 / 1440

E-mail address: sfeyo@fe.up.pt (S. F.de Azevedo).
}

model. This type of solution may be most advantageous in terms of benefits for process operation.

Over the past 20 years different approaches have been proposed for estimation of growth kinetics in bioprocesses. Most of them are model-based estimators using the general theoretical framework developed by Bastin and Dochain [4]. Farza and co-authors [5-8] have proposed observer-based estimators (OBE) whose fundamentals are found in the nonlinear systems theory. A discrete-time version of these OBE was also introduced using direct Euler discretization [9]. These works estimate reaction rates from the measurement of state variable concentrations. Some authors have been using exit-gas analysis such as oxygen transfer rate and carbon dioxide transfer rate measurements for the estimation of the growth rates [10,11]. More recently, Perrier et al. [12] studied in detail the tuning of OBE for on-line estimation of kinetic parameters.

Though, in general terms, the theory and scope of applications of OBE are well documented in the literature, several issues concerning stability and dynamics of convergence related to the tuning of design parameters remain to be studied. Such issues are discussed in detail in the present paper.

The paper is organised in the following way. In Section 2 we review the concept of observer-based reaction 


\begin{tabular}{|c|c|c|c|}
\hline \multicolumn{2}{|c|}{ Nomenclature } & \multirow{2}{*}{$\begin{array}{l}\mu_{s}^{o} \\
\hat{\rho}\end{array}$} & \multirow{2}{*}{$\begin{array}{l}\text { specific growth rate for the respiratory } \\
\text { growth on glucose pathway } \\
\text { vector of } \rho(t) \text { estimates }\end{array}$} \\
\hline C & dissolved oxygen concentration & & \\
\hline$C T R$ & carbon dioxide transfer rate & $\rho(t)$ & vector of completely unknown time-varying \\
\hline$D$ & dilution rate & & parameters \\
\hline E & ethanol concentration & $\tau_{i}$ & natural period of oscillation \\
\hline$F$ & vector of mass feed rates & $\Omega$ & gain matrix \\
\hline$F_{\text {in }}$ & influent flow rate & $\omega_{i}$ & diagonal elements of $\Omega$ \\
\hline G & dissolved carbon dioxide concentration & $\xi$ & vector of state variables \\
\hline$\Phi(\xi)$ & $(m \times m)$ matrix of known functions of the & $\hat{\xi}$ & vector of estimated states \\
\hline & state & $\xi_{1}$ & vector of measured state variables \\
\hline K & yield coefficients matrix & $\xi_{2}$ & vector of non-measured state variables \\
\hline$k_{\mathrm{i}}$ & yield coefficient & $\hat{\xi}_{2}$ & vector of estimated nonmeasured state vari- \\
\hline OTR & oxygen transfer rate & & ables \\
\hline$Q$ & vector of mass outflow in gaseous form & $\psi$ & vector of transformed state variables \\
\hline$\hat{S}$ & glucose concentration & $\zeta_{i}$ & damping coefficient \\
\hline$S_{\text {in }}$ & glucose concentration in the feed & & \\
\hline & sampling period & \multicolumn{2}{|c|}{ Mathematical notations } \\
\hline$X$ & biomass concentration & $\sup$ & $\begin{array}{l}\text { supremum } \\
\text { minimum }\end{array}$ \\
\hline \multicolumn{2}{|c|}{ Greek letters } & $\max$ & maximum \\
\hline$\Gamma$ & gain matrix & $\operatorname{diag}\{\}$. & diagonal matrix \\
\hline$\gamma_{i}$ & diagonal elements of $\Gamma$ & \multirow{2}{*}{\multicolumn{2}{|c|}{ Abbreviations }} \\
\hline$\varphi$ & vector of reaction rates & & \\
\hline$\mu$ & vector of specific growth rates & BIBO & bounded input bounded output \\
\hline$\hat{\mu}$ & vector of estimated specific growth rates & LTV & linear time variant \\
\hline$\mu_{e}^{o}$ & specific growth rate for the respiratory & OBE & observer-based estimator \\
\hline & growth on ethanol pathway & SODE & second order dynamics estimator \\
\hline$\mu_{s}^{r}$ & $\begin{array}{l}\text { specific growth rate for the fermentative } \\
\text { growth on glucose pathway }\end{array}$ & & \\
\hline
\end{tabular}

kinetics estimator. In Section 3 we present a detailed analysis of stability and dynamics of convergence. A tuning strategy is derived, which aims at imposing quasiinvariant second-order dynamics of convergence and the proof of global stability is presented. In Section 4 we develop a state and reaction rates estimation scheme for a Baker's yeast fed-batch production process, employing the algorithms of Sections 2 and 3. In Section 4 we present the main results obtained with the estimation scheme of Section 3 with a simulated experiment. Finally, in Section 5, we present the main conclusions.

\section{The observer-based estimator}

\subsection{The general case}

Bastin and Dochain [4] proposed a methodology for state and parameter estimation based upon a general dynamical model for stirred-tank reactors (although the original formulation was applied exclusively to stirredtank bioreactors, it can be equally applied to chemical reactors as shown in [13]): $\frac{\mathrm{d} \xi}{\mathrm{d} t}=K \varphi(\xi)-D \xi+F-Q$

where $\xi$ is the state vector (the set of $n$ component concentrations), $\varphi(\xi)$ is the $m \times 1$ vector of reaction rates, $K$ the $n \times m$ yield coefficients matrix, $D$ the dilution rate (a scalar), $F$ the $n \times 1$ feed rate vector and $Q$ the $n \times 1$ gaseous outflow rate vector.

In Eq. (1) the reaction rates $\varphi(\xi)$ are defined as:

$\varphi_{i}(\xi)=h_{i}(\xi) \rho_{i}(\xi) \quad i=1, \ldots, m$

where $h_{i}(\xi)$ is a known function of the state while $\rho_{i}(\xi)$ is an unknown function of the state. Or, more generally:

$\varphi(\xi)=\Phi(\xi) \rho(\xi)$

with $\Phi(\xi)=\operatorname{diag}\left(\left[h_{1}(\xi) \cdots h_{m}(\xi)\right]\right)$ and

$$
\rho(\xi)=\left[\rho_{1}(\xi) \cdots \rho_{m}(\xi)\right]^{T}
$$

The strategy is to lump in $\Phi(\xi)$ all the prior knowledge regarding the kinetics and to consider $\rho(\xi)$ as a 
completely unknown time-varying parameter that can be estimated on-line through the use of parameter estimators.

Based on this generic dynamic model, the observerbased estimator (OBE) is derived with the goal of estimating on-line the unknown vector $\rho(\xi)$ from the online knowledge of all state variables when the yield coefficients are known and constant. When only a limited number of state variables are effectively measured on-line, then the estimator can run simultaneously with a state estimator that provides the unmeasured states. The estimator is stated as:

$$
\begin{aligned}
& \frac{\mathrm{d} \hat{\xi}}{\mathrm{d} t}=K \Phi(\xi) \hat{\rho}-D \xi+F-Q+\Omega(\xi-\hat{\xi}) \\
& \frac{\mathrm{d} \hat{\rho}}{\mathrm{d} t}=[K \Phi(\xi)]^{T} \Gamma(\xi-\hat{\xi})
\end{aligned}
$$

where $\hat{\xi}$ is an estimate of $\xi, \hat{\rho}$ is an estimate of $\rho, \Omega$ and $\Gamma$ are square $n \times n$ matrices of design parameters available to the user to control the stability and the tracking properties of the algorithm.

The stability conditions were established in [4] and may be summarised as follows:

C1. state variables $\xi$ are positive and bounded for all $t$.

C2. $\rho(\xi)$ is a differentiable function of $\xi$.

C3. $\Omega$ is a $n \times n$ constant matrix with all its eigenvalues having strictly real parts.

C4. $\Gamma$ is a $n \times n$ constant matrix such that the matrix $\boldsymbol{\Omega}^{\mathrm{T}} \boldsymbol{\Gamma}+\boldsymbol{\Gamma}^{\mathrm{T}} \boldsymbol{\Omega}$ is positive definite.

$\mathrm{C} 5 . \mathrm{K} \Phi(\xi)$ is a persistently exciting matrix.

Conditions $\mathrm{C} 3$ and $\mathrm{C} 4$ define some restrictions to the tuning of the design parameters in matrices $\Omega$ and $\Gamma$ To some extent the user may control the stability and the tracking properties of the algorithm through the proper setting of these parameters. By defining:

$\boldsymbol{\Omega}=\operatorname{diag}\left\{\left[\omega_{1} \ldots \omega_{n}\right]\right\} \quad \boldsymbol{\Gamma}=\operatorname{diag}\left\{\left[\gamma_{1} \ldots \gamma_{n}\right]\right\}$

being $\omega_{i}$ and $\gamma_{i} 2 \times n$ strictly positive real constants then conditions $\mathrm{C} 3$ and $\mathrm{C} 4$ are automatically verified and the tuning procedure reduces to the calibration by trial and error of $2 \times n$ scalar constants. Notice also that while condition $\mathrm{C} 1$ is acceptable in most chemical and biochemical applications since most often state variables are concentrations, condition C5 must be properly tested before application.

With such a strategy stability is guaranteed but the user has few control on the tracking properties of the system as it will be shown below in Section 5. The relationships between stability and tracking properties can however be better characterised in a more restricted class of problems: (i) when the reaction term can be expressed by Eq. (2); and (ii) when a subset of the state space equations is enough for designing the observerbased estimator (which is then called reduced order observer-based estimator). Fortunately this situation occurs quite often in biochemical applications. We develop further this particular case in the following sections.

\subsection{The reduced-order observer-based estimator}

The observer-based estimator (4) is based on the full dynamical model of the process. In practice this is not always necessary. In many applications it is sufficient to design the estimator from a subset of the state equations provided they involve all the parameters that need to be estimated. This reduced-order OBE is based on the following assumptions and corresponding developments:

A1. There are $m$ parameters which need to be estimated.

A2. There is a subset of $m$ equations of the full state space model that involves all the $m$ parameters which need to be estimated:

$\frac{\mathrm{d} \xi_{a}}{\mathrm{~d} t}=K_{a} \Phi(\xi) \rho(\xi)-D \xi_{a}+F_{a}-Q_{a}$.

A3. In Eq. (6) $K_{a}$ is a $m \times m$ full-rank matrix By considering the transformation:

$\psi=K_{a}^{-1} \xi_{a}$

then Eq. (6) can be re-written as:

$\frac{\mathrm{d} \psi}{\mathrm{d} t}=\Phi(\xi) \rho(\xi)-D \psi+K_{a}^{-1}\left(F_{a}-Q_{a}\right)$.

With the reaction rates definitions in Eqs. (1) and (2), $\Phi(\xi)$ is a $m \times m$ diagonal matrix and Eq. (8) can be reformulated in a decoupled format,

$\frac{\mathrm{d} \psi_{i}}{\mathrm{~d} t}=h_{i}(\xi) \rho_{i}(\xi)-D \psi_{i}+u_{i}$

$u_{i}$ being the $i$ th element of vector $U=K_{a}^{-1}\left(F_{a}-Q_{a}\right)$.

Based on the reformulated process model (9), the reduced-order $O B E$ is written as:

$\frac{\mathrm{d} \hat{\psi}_{i}}{\mathrm{~d} t}=h_{i}(\xi) \hat{\rho}_{i}-D \psi_{i}+u_{i}+\omega_{i}\left(\psi_{i}-\hat{\psi}_{i}\right)$

$\frac{\mathrm{d} \hat{\rho}_{i}}{\mathrm{~d} t}=h_{i}(\xi) \gamma_{i}\left(\psi_{i}-\hat{\psi}_{i}\right)$

Pomerleau and Perrier [14] suggested a pole placement technique for tuning the design parameters $\omega_{i}$ and 
$\gamma_{i}$ in the discrete time formulation of estimator (10). Oliveira et al. [15] analysed in a previous paper the continuous-time formulation of estimator (10) showing that defining constant values for the design parameters the dynamics of convergence of estimated parameters to 'true' parameters follows second order dynamics with time-varying characteristic coefficients (time constant and damping factor).

In order to better control the dynamics of convergence of the OBE estimator, recently Perrier et al. [12] suggested a re-definition of the regressor in the updating law of $\hat{\rho}$ [Eq. (10b)] as:

$\frac{\mathrm{d} \hat{\psi}_{i}}{\mathrm{~d} t}=h_{i}(\xi) \hat{\rho}_{i}-D \psi_{i}+u_{i}+\omega_{i}\left(\psi_{i}-\hat{\psi}_{i}\right)$

$\frac{\mathrm{d} \hat{\rho}_{i}}{\mathrm{~d} t}=\frac{\gamma_{i}}{h_{i}(\xi)}\left(\psi_{i}-\hat{\psi}_{i}\right)$.

where $\omega_{i}$ and $\gamma_{i}$ are positive real constants used for calibrating the estimator. Still, in both continuous and discrete systems, the relationships between stability, dynamics of convergence and tuning of design parameters are open for analysis as it will be now discussed.

\section{A detailed analysis of stability, dynamics of convergence and tuning of design parameters}

\subsection{Derivation of stability conditions}

The error system of estimator (11) can be shown to be a second order linear time variant (LTV) system:

$\frac{\mathrm{d}}{\mathrm{d} t}\left[\begin{array}{c}\tilde{\psi}_{i} \\ \tilde{\rho}_{i}\end{array}\right]=\left[\begin{array}{ll}-\omega_{i} & h_{i}(\xi) \\ -\gamma_{i} h_{i}^{-1}(\xi) & 0\end{array}\right]\left[\begin{array}{c}\tilde{\psi}_{i} \\ \tilde{\rho}_{i}\end{array}\right]+\left[\begin{array}{l}0 \\ \mathrm{~d} \rho_{i} \\ \mathrm{~d} t\end{array}\right]$

with $\tilde{\psi}_{i}=\psi_{i}-\hat{\psi}_{i}, \quad \tilde{\rho}_{i}=\rho_{i}-\hat{\rho}_{i}, \quad i=1, \ldots, m$

where $\mathrm{d} \rho_{i} / \mathrm{d} t$ is considered as an external persistent disturbance.

It is a standard result of the BIBO stability theory that a LTV system perturbed by an external disturbance is globally stable if the unperturbed system is uniformly asymptotically stable and the disturbance vector is bounded [16].

Stability conditions $\mathrm{C} 1$ and $\mathrm{C} 2$ (see Section 2.1) ensure the boundness of $\mathrm{d} \rho_{i} / \mathrm{d} t$ [4]. Still, it remains to be proved that the unforced system is uniformly asymptotically stable.

Let us first reformulate the error system (12) and concentrate our attention on the unforced system:

Considering the transformation $y=\tilde{\psi} / h$ (to simplify the present analysis the index ' $i$ ' will be omitted hereafter), the set of Eqs. (12) results in, $\frac{\mathrm{d} y}{\mathrm{~d} t}=-a(t) y+\tilde{\rho}$

$\frac{\mathrm{d} \tilde{\rho}}{\mathrm{d} t}=-\gamma y+\frac{\mathrm{d} \rho}{\mathrm{d} t}$

where

$a(t)=\omega(t)+\frac{\mathrm{d} \ln (h)}{\mathrm{d} t}$

Choosing the following candidate Lyapunov function:

$V(y, \tilde{\rho})=\gamma y^{2}+\tilde{\rho}^{2}$

whose time derivative along the solution of system (13) is given by:

$\frac{\mathrm{d} V}{\mathrm{~d} t}=-2 a(t) y^{2}=-E^{T} Q(t) E$

where

$E^{T}=\left[\begin{array}{ll}y & \tilde{\rho}\end{array}\right], \quad Q(t)=\left[\begin{array}{ll}a(t) & 0 \\ 0 & 0\end{array}\right]$

it follows that if $a(t) \geqslant 0 \forall_{t \geqslant t_{0}}$ then $Q(t)$ is positive semidefinite. Hence the equilibrium state $E=0$ is uniformly stable [16].

Supposing that stability conditions $\mathrm{C} 1$ and $\mathrm{C} 2$ hold, the further conditions under which $a(t) \geqslant 0 \quad \forall_{t \geqslant t_{0}}$ are stated as:

C6. $h(\xi)$ is a differentiable function of $\xi$, which means that $|\dot{h}|$ is bounded

C7. $h(\xi)$ is bounded as follows: $0<h_{\min } \leqslant h_{i}(\xi) \leqslant h_{\max }$ $\forall t \geqslant t_{0}$

C8. $\omega(t) \geqslant-\frac{\mathrm{d} \ln h}{\mathrm{~d} t} \forall_{t \geqslant t_{0}}$

Still, since $Q(t)$ is positive semi-definite and timevarying, it cannot be concluded that system (13) is uniformly asymptotically stable.

In the lines bellow, the exponential stability of system (13) is proved. A qualitative outline of the proof can be given in two steps:

(i) In system (13) $y(t)$ has to assume a large value at some instance in every interval $\left[t, t+T_{o}\right]$;

(ii) Since $\dot{V}$ is as given by Eq. (16), this implies that $V(t)$ decreases over every interval of length $T_{o}$, which ensures uniform asymptotic stability.

Proof. (i) Start by assuming that $\left|y_{i}(t)\right|<\alpha\left\|E_{i}(t)\right\|$ $\forall t \in\left[t_{o}, t_{o}+T_{o}\right]$, where $\alpha \in[0,1]$.

Integrating Eq. (13a) over the time interval $\left[t_{2}, t_{2}+\delta_{0}\right] \subset\left[t_{0}, t_{0}+T_{0}\right]$, it follows that 
$\left|y\left(t_{2}+\delta_{0}\right)\right|+\left|y\left(t_{2}\right)\right| \geqslant \int_{t_{2}}^{t_{2}+\delta_{0}}|\tilde{\rho}(\tau)| d \tau-a_{\max } \int_{t_{2}}^{t_{2}+\delta_{0}}|y(\tau)| \mathrm{d} \tau$

where $a_{\max }$ is the maximum value of $|a(t)|$. Therefore

$$
\begin{aligned}
& \left|y\left(t_{2}+\delta_{0}\right)\right| \geqslant \int_{t_{2}}^{t_{2}+\delta_{0}}|\tilde{\rho}(\tau)| d \tau \\
& -\left(\delta_{0} a_{\max }+1\right) \sup _{\tau \in\left[t_{2}, t_{2}+\delta_{0}\right]}|y(\tau)|
\end{aligned}
$$

since $\left|y\left(t_{2}\right)\right|$ is always less then $\sup |y(\tau)|$ in $\tau \in\left[t_{2}, t_{2}+\delta_{0}\right]$. On the other hand

$\int_{t_{2}}^{t_{2}+\delta_{0}}|\tilde{\rho}(\tau)| \mathrm{d} \tau \geqslant \int_{t_{2}}^{t_{2}+\delta_{0}}\left|\tilde{\rho}\left(t_{2}\right)\right| \mathrm{d} \tau-\int_{t_{2}}^{t_{2}+\delta_{0}}\left|\tilde{\rho}\left(t_{2}\right)-\tilde{\rho}(\tau)\right| \mathrm{d} \tau$

$\geqslant \delta_{0}\left|\tilde{\rho}\left(t_{2}\right)\right|-\delta_{0} \sup _{\tau \in\left[t_{2}, t_{2}+\delta_{0}\right]}\left|\tilde{\rho}\left(t_{2}\right)-\tilde{\rho}(\tau)\right|$

$\geqslant \delta_{0}\left|\tilde{\rho}\left(t_{2}\right)\right|-\delta_{0} \int_{t_{2}}^{t_{2}+\delta_{0}}|\dot{\tilde{\rho}}| \mathrm{d} \tau$

since the distance between to points $\tilde{\rho}\left(t_{2}\right)$ and $\tilde{\rho}(\tau)$ is always less then the arc length $\int_{t_{2}}^{t_{2}+\delta_{0}}|\dot{\tilde{\rho}}| \mathrm{d} \tau$.

Hence, evaluating $\dot{\tilde{\rho}}$ from Eq. (13b), Eq. (19) becomes

$\int_{t_{2}}^{t_{2}+\delta_{0}}|\tilde{\rho}(\tau)| \mathrm{d} \tau \geqslant \delta_{0}\left|\tilde{\rho}\left(t_{2}\right)\right|-b \sup _{\tau \in\left[t_{2}, t_{2}+\delta_{0}\right]}|y(\tau)|$

where $b=\gamma \delta_{0}^{2}+a_{\max } \delta_{0}+1$.

From the initial supposition regarding $|y(t)|$, it follows that $\sup |y(\tau)|$ in $\tau \in\left[t_{2}, t_{2}+\delta_{0}\right]$ is always less then $\alpha\left\|E\left(t_{2}+\delta_{0}\right)\right\|$, and also $\left|\tilde{\rho}\left(t_{2}\right)\right|$ is always larger then $\sqrt{1-\alpha^{2}}\left\|E\left(t_{2}\right)\right\|$

Since $\frac{\mathrm{d}\|E(t)\|}{\mathrm{d} t} \leqslant \frac{-a(t) y^{2}}{\min (\gamma, 1)} \leqslant 0 \quad \forall t \geqslant t_{0}$, then $\|E(t 2)\| \geqslant$ $\left\|E\left(t_{2}+\delta_{0}\right)\right\|$. Hence Eq. (20) becomes

$\left|y\left(t_{2}+\delta_{0}\right)\right| \geqslant\left(\delta_{0} \sqrt{1-\alpha^{2}}-b \alpha\right)\left\|E\left(t_{2}+\delta_{0}\right)\right\|$.

\section{Choosing}

$\alpha^{2}=\frac{\delta_{0}^{2}}{\delta_{0}^{2}+(1+b)^{2}}$

then Eq. (21) becomes

$\left|y\left(t_{2}+\delta_{0}\right)\right| \geqslant \alpha\left\|E\left(t_{2}+\delta_{0}\right)\right\|$

which is a contradiction of the initial assumption regarding $|y(t)|$. Point $(i)$ is, therefore, proved.

(ii) Analysis of the trajectory $V(t)$

Let us now integrate Eq. (16) over a time interval $\left[t_{1}, t_{1}+T\right] \subset\left[t_{0}, t_{0}+T_{0}\right]$
$V\left(t_{1}\right)-V\left(t_{1}+T\right) \geqslant 2 a_{\max } \int_{t_{1}}^{t_{1}+T}|y(\tau)|^{2} \mathrm{~d} \tau$

and by the Cauchy-Schwarz inequality

$V\left(t_{1}\right)-V\left(t_{1}+T\right) \geqslant \frac{2 a_{\max }}{T}\left(\int_{t_{1}}^{t_{1}+T}|y(\tau)| \mathrm{d} \tau\right)^{2}$

Further, by considering that

$$
\begin{aligned}
& \int_{t_{1}}^{t_{1}+T}|y(\tau)| \mathrm{d} \tau \geqslant \int_{t_{1}}^{t_{1}+T}\left|y\left(t_{1}\right)\right| \mathrm{d} \tau-\int_{t_{2}}^{t_{2}+\delta_{0}}|\dot{y}| \mathrm{d} \tau \\
& \geqslant T\left|y\left(t_{1}\right)\right|-T^{2} d\left\|E\left(t_{1}\right)\right\|
\end{aligned}
$$

where $d=a_{\max }+1$, then choosing $t_{1}=t_{2}+\delta_{0}$ we have that

$\int_{t_{1}}^{t_{1}+T}|y(\tau)| \mathrm{d} \tau \geqslant T(\alpha-T d)\left\|E\left(t_{1}\right)\right\|$.

Hence Eq. (25) becomes

$V\left(t_{1}\right)-V\left(t_{1}+T\right) \geqslant 2 \frac{a_{\max }}{\mathrm{d}} T d(\alpha-T d)^{2}\left\|E\left(t_{1}\right)\right\|^{2}$

being $T$ less than $\alpha / d$.

Since $\|E(t)\|^{2}$ is always larger then $V(t) / \max (\gamma, 1)$, then choosing $T=\min \left(t_{0}+T_{0}-t_{1}, \alpha / d\right)$ we conclude that

$V\left(t_{0}+T_{0}\right) \leqslant V\left(t_{1}+T\right) \leqslant(1-\beta) V\left(t_{1}\right) \leqslant(1-\beta) V\left(t_{0}\right)$

where

$\beta=\frac{2 a_{\max }}{\max (\gamma, 1) d} T d(\alpha-T d)^{2}$

Since $a_{\max } / d$ is always less then 1 and $T=\alpha /(3 d)$ is a maximum point of the function $f(T)=T d(\alpha-T d)^{2}$, we conclude that

$\beta \in\left[0, \frac{8 \alpha^{3}}{27 \max (\gamma, 1)}\right] \subset[0,1]$

Hence, the final conclusion can be taken that the unperturbed estimator (13) is uniformly asymptotically stable, thus system (13) is globally stable provided conditions $\mathrm{C} 1, \mathrm{C} 2$ and $\mathrm{C} 6-\mathrm{C} 8$ hold true.

It should be noted that condition $\mathrm{C} 8$ is obeyed depending on the choice of design parameters. This is discussed next. 


\subsection{Dynamics of convergence, stability conditions, and tuning}

The above derived stability conditions impose some constraints on the choice of the design parameters. Additionally and if possible the choice of design parameters should be easily related to the type of dynamics of convergence, which one might wish to impose to the estimator. This issue will be addressed in the present section (again, to simplify the reading index ' $i$ ' in set of Eqs. (11) will be omitted).

Differentiating Eq. (11b) gives

$\frac{\mathrm{d}^{2} \hat{\rho}}{\mathrm{d} t^{2}}=\frac{\gamma}{h} \frac{\mathrm{d} \tilde{\psi}}{\mathrm{d} t}-\gamma \frac{1}{h^{2}} \frac{\mathrm{d} h}{\mathrm{~d} t} \tilde{\psi}$

Combining Eqs. (32) and (11b) and the first equation in the error system (12), it follows that

$\tau^{2} \frac{\mathrm{d}^{2} \hat{\rho}}{\mathrm{d} t^{2}}+2 \zeta \tau \frac{\mathrm{d} \hat{\rho}}{\mathrm{d} t}+\hat{\rho}=\rho$

with $\tau$ and $\zeta$ given by

$\tau=\gamma^{-0.5}$

$\zeta=\frac{\tau}{2}\left(\omega+\frac{\mathrm{d} \ln h}{\mathrm{~d} t}\right)$

Eq. (33) shows that kinetic parameters $\hat{\rho}(t)$ may be led to converge to their true value $\rho(t)$ with a second order dynamic response with constant natural period of oscillation $\tau$ and time-varying damping coefficient $\zeta(t)$. From condition $\mathrm{C} 8$ it was concluded that $\omega$ may be timevarying but must be always larger then the value of$\mathrm{d}(\ln h) / \mathrm{d} t$. Supposing that the term

$\phi=\frac{\mathrm{d} \ln h(\xi)}{\mathrm{d} t}$

is known on-line corrupted by an error $\varepsilon(t)$ (in practice the on-line knowledge of $\phi$ requires an approximation to the time derivative $\mathrm{d} \ln (h) / \mathrm{d} t$, and, therefore, the estimated value $\hat{\phi}$ is always corrupted by the approximation error $\varepsilon(t)=\hat{\phi}-\phi)$ and defining $\gamma$ and $\omega(t)$ as:

$\gamma=\frac{1}{\tau^{2}}$

$\omega(t)=2 \zeta_{d} / \tau-\hat{\phi}=2 \zeta_{d} / \tau-\phi-\varepsilon(t)$

then the damping coefficient $\zeta(t)$ is time-varying and related to the desired value $\zeta_{d}$ and to the approximation error $\varepsilon(t)$ in the following way: $\zeta(t)=\zeta_{d}\left(1-\frac{\tau \varepsilon(t)}{2 \zeta_{d}}\right)$.

With this strategy, the time dependency of $\zeta$ is to some extent reduced. However care should be taken when the measured or the estimated state variables are noisy. In this situation the numerical differentiation of $\phi$ may have large errors $\epsilon$ attached.

Notice that defining $\omega(t)$ by Eq. (36b) implies that condition C8 which states that $\omega(t)>-\phi(\xi) \forall_{t}$ is verified if

$\frac{2 \zeta_{d}}{\tau}>\varepsilon(t), \quad \forall_{t}$

Notice also, and finally, that as given by Eq. (37) this is equivalent to state that $\zeta(t)$ must be positive for all $t$.

Hereafter the estimator constituted by the set of Eqs. (11), together with the tuning Eqs. (36) and (37), leading to a second order dynamics of convergence will be termed as SODE - second order dynamics estimator.

\subsection{Numerical implementation, stability condition and tuning}

The numerical implementation of estimator (11) requires a discrete-time formulation. The switch from the continuous-time equations to the discrete time versions leads to specific stability problems, in which the integration step $T$ plays an important role. The simplest approach, often employed, is Euler's discretisation of the continuous time equations. The implications in the stability of using such an Euler discretisation are now analysed:

A forward Euler discretisation of Eqs. (11) with $\omega(t)$ and $\gamma$ given by Eqs. (36) and (37) results in the following discrete-time equations:

$$
\begin{aligned}
\hat{\psi}_{t+1}= & \hat{\psi}_{t}-T D_{t} \psi_{t}+T h_{t} \hat{\rho}_{t}+T U_{t} \\
& +T \omega_{t}\left(\psi_{t}-\hat{\psi}_{t}\right)
\end{aligned}
$$

$\hat{\rho}_{t+1}=\hat{\rho}_{t}+\frac{T}{\tau^{2} h_{t}}\left(\psi_{t}-\hat{\psi}_{t}\right)$

with

$\omega_{t}=\frac{2 \zeta}{\tau}-\frac{\ln \left(h_{t+1}\right)-\ln \left(h_{t}\right)}{T}$.

The discrete error system is as follows

$\tilde{\psi}_{t+1}=\left(1-T \omega_{t}\right) \tilde{\psi}_{t}+T h_{t} \tilde{\rho}_{t}$

$\tilde{\rho}_{t+1}=-\frac{T}{\tau^{2} h_{t}} \tilde{\psi}_{t}+\tilde{\rho}_{t}+\left(\rho_{t+1}-\rho_{t}\right)$ 
which is equivalent to

$E_{t+1}=A E_{t}+B_{t}$

with

$$
\begin{gathered}
E_{t}=\left[\begin{array}{c}
\tilde{\psi}_{t} \\
h_{t} \\
\tilde{\rho}_{t}
\end{array}\right] \quad A=\left[\begin{array}{ll}
1-\frac{2 \zeta}{\tau} T & T \\
-\frac{T}{\tau^{2}} & 1
\end{array}\right] \\
B_{t}=\left[\begin{array}{l}
0 \\
\rho_{t+1}-\rho_{t}
\end{array}\right] .
\end{gathered}
$$

The discrete-time error system (43) is linear timeinvariant. The unforced system is exponentially stable [hence the output error of system (43) is bounded] if the eigenvalues of matrix $A$ stay inside the unit circle. The eigenvalues of matrix $A$ and the stability condition in terms of integration step are shown in Table 1. The analysis of such eigenvalues leads to the conclusion that the range allowed for the integration step $T$ is bounded and conditioned by the choice of $\tau$ and $\zeta$. Notice also that these restrictions are typical of a second order dynamics system and would be the same if the analysis had been carried out from the discrete version of Eq. (33).

\section{Results and discussion}

The results and conclusions of the preceding sections are now illustrated with an application to a baker's yeast cultivation process. The process data were obtained by simulation, using the dynamical model for the fed-batch fermenter described by Feyo de Azevedo et al. [17].

Process model and operating conditions: the mass balances to biomass, substrate, ethanol, oxygen and carbon dioxide, in terms of concentration in the fermentation media, take the matrix form:

$$
\begin{aligned}
\frac{\mathrm{d}}{\mathrm{d} t}\left[\begin{array}{c}
X \\
S \\
E \\
C \\
G
\end{array}\right]= & {\left[\begin{array}{ccc}
1 & 1 & 1 \\
-k_{1} & -k_{2} & 0 \\
0 & k_{3} & -k_{4} \\
-k_{5} & 0 & -k_{6} \\
k_{7} & k_{8} & k_{9}
\end{array}\right]\left[\begin{array}{c}
\mu_{s}^{o} \\
\mu_{s}^{r} \\
\mu_{e}^{o}
\end{array}\right] X-D\left[\begin{array}{c}
X \\
S \\
E \\
C \\
G
\end{array}\right] } \\
+ & {\left[\begin{array}{c}
0 \\
D S_{i n} \\
0 \\
O T R \\
-C T R
\end{array}\right] }
\end{aligned}
$$

where $k_{i}$ are the yield coefficients, $O T R$ is the oxygen transfer rate, $C T R$ is the carbon dioxide transfer rate, $D$ is the dilution rate defined as $D=F_{\text {in }} / V, V$ is the broth volume and $F_{\text {in }}$ is the influent flow rate. The oxygen
Table 1

Eigenvalues of the discrete-time formulation error system and stability condition for integration step

\begin{tabular}{llll}
\hline & $\zeta<1$ & $\zeta=1$ & $\zeta>1$ \\
\hline$\lambda_{1}$ & $1-\frac{T}{\tau}\left(\zeta+i \sqrt{1-\zeta^{2}}\right)$ & $1-\frac{T}{\tau}$ & $1-\frac{T}{\tau}\left(\zeta+\sqrt{\zeta^{2}-1}\right)$ \\
$\lambda_{2}$ & $1-\frac{T}{\tau}\left(\zeta-i \sqrt{1-\zeta^{2}}\right)$ & $1-\frac{T}{\tau}$ & $1-\frac{T}{\tau}\left(\zeta-\sqrt{\zeta^{2}-1}\right)$ \\
$T$ & $0<T<2 \zeta \tau$ & $0<T<\tau$ & $0<T<\frac{\tau}{\zeta+\sqrt{\zeta^{2}-1}}$ \\
\hline
\end{tabular}

transfer rate is computed as $O T R=K_{L} a\left(C^{*}-C\right)$ where $K_{L} a$ is the global mass transfer coefficient and $C^{*}$ the equilibrium concentration of dissolved oxygen. The carbon dioxide transfer rate is computed as $C T R=K_{V} K_{L} a G$. The value for $K_{L} a, K_{v}$ and $C^{*}$ were assumed $100 \mathrm{~h}^{-1}, 0.2$ and $0.007 \mathrm{~g} / 1$ respectively. The bottleneck kinetic model of Sonnleitner and Käppeli [18] was assumed together with values for the yield coefficients from [14]. The process was simulated with the following initial conditions:

$$
\begin{aligned}
& X(0)=1.0 \mathrm{~g} / 1, \quad S(0)=0.02 \mathrm{~g} / 1, \\
& E(0)=0.15 \mathrm{~g} / 1, \quad C(0)=0.0066 \mathrm{~g} / 1, \\
& G(0)=0.008 \mathrm{~g} / 1, \quad V(0)=3.51
\end{aligned}
$$

The simulation results for an $18 \mathrm{~h}$ cultivation are plotted in Figs. 1a-c and 2. Fig. 1a shows the feed rate profile imposed to the process. The profiles of the gaseous outflow rates $O T R$ and $C T R$, and of the state variables are plotted in Fig. $1 \mathrm{~b}$ and $\mathrm{c}$, respectively. In Fig. 2 the corresponding specific growth rate profiles given by the kinetic model adopted are plotted.

As shown in Fig. 2 the specific growth rates related to ethanol production and consumption $\left(\mu_{s}^{r}\right.$ and $\mu_{e}^{o}$ respectively) switch alternatively from zero to positive values and are not at any instant simultaneously positive. This illustrates the two metabolic states - respirofermentative and respirative- reported in [18]. The input feed rate profile (Fig. 1a) was chosen intentionally to promote this switching a considerable number of times. For the present conditions the switching between respiro-fermentative and respirative metabolic states occurred seven times. Fig. 2 is divided in several regions distinguishing the two different process states: respiro-fermentative (RF) with ethanol production, and respirative $(\mathrm{R})$ with ethanol consumption. The curves shown in Fig. 2 are taken as the 'true' specific growth rate profiles, which will be the target parameters. These three specific growth rates are treated as three unknown process variables that must be estimated, using the estimators previously described. As 
(a)

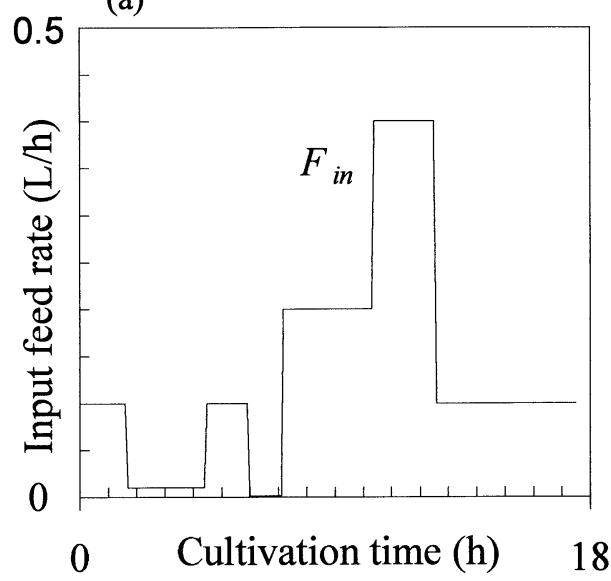

(b)

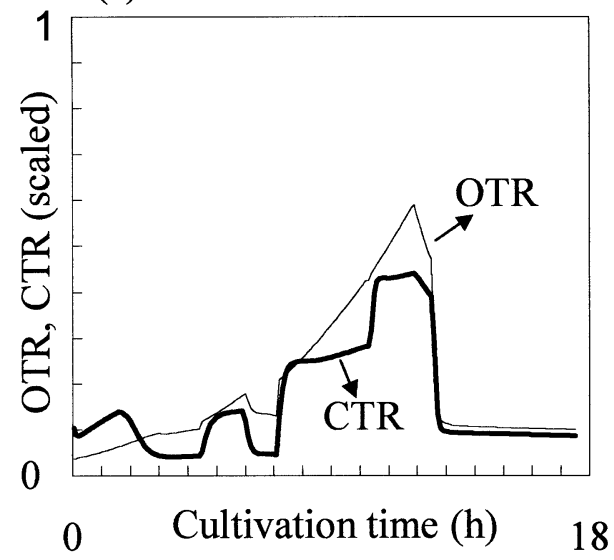

(c)

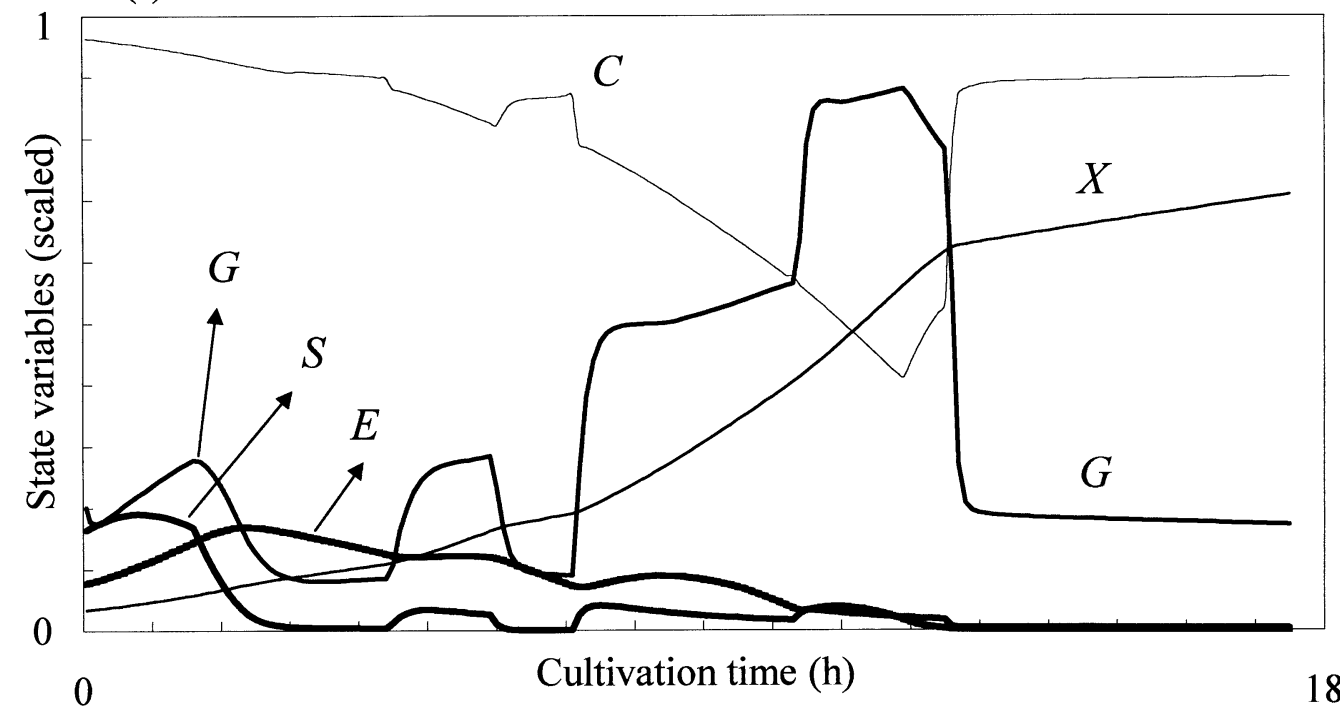

Fig. 1. Operating data for Baker's yeast fermentation: (a) input feed rate $F_{\text {in }}(0.0-0.5 \mathrm{l} / \mathrm{h})$, glucose concentration on the feed $S_{\text {in }}(250 \mathrm{~g} / \mathrm{l})$ and volume $V(0.0-10.01)$. (b) Gaseous transfer rates: oxygen transfer rate $O T R\left(0.0-7.0 \mathrm{~g} \mathrm{l}^{-1} \mathrm{~h}^{-1}\right)$ and carbon dioxide transfer rate $C T R\left(0.0-12.0 \mathrm{~g} \mathrm{l}^{-1} \mathrm{~h}^{-1}\right)$. (c) State variables: biomass $X(0.0-30.0 \mathrm{~g} / \mathrm{l})$, glucose $S(0.0-1.25 \mathrm{~g} / \mathrm{l})$, ethanol $E(0.0-2.0 \mathrm{~g} / \mathrm{l})$, oxygen $C(0.0-0.007 \mathrm{~g} / \mathrm{l})$ and carbon dioxide $G(0.0-0.03 \mathrm{~g} / \mathrm{l})$.

such, the functions $\Phi(\xi)$ and $\rho(\xi)$ in Eq. (3) are defined in the following way:

$\Phi(\xi)=\operatorname{diag}(X)$ and $\rho^{T}(\xi)=\left[\begin{array}{lll}\mu_{s}^{o} & \mu_{s}^{r} & \mu_{e}^{o}\end{array}\right]$.

\subsection{Reduced-order OBE kinetics estimator}

Some practical aspects reported in previous works for OBE design [14] have been adopted here, namely: (i) the state space equations for oxygen and carbon dioxide are employed since these variables are easily accessible online; and (ii) the design assumes the existence of two partial models corresponding to the two metabolic states (respiro-fermentative and respirative) yielding to partial algorithms. This latter strategy is used to prevent problems related to the inversion of matrix $K_{a}$ in Eqs. (7) and (8), which arise when the design is based on the full dynamical model. The switch between partial algorithms is detected by the transition between positive to negative values of the growth rate estimate related to ethanol production or consumption. The resulting equations of the reduced-order OBE are:

For the respiro-fermentative state:

$\psi=\left[\begin{array}{ll}-k_{5} & 0 \\ k_{7} & k_{8}\end{array}\right]^{-1}\left[\begin{array}{l}C \\ G\end{array}\right]$ 


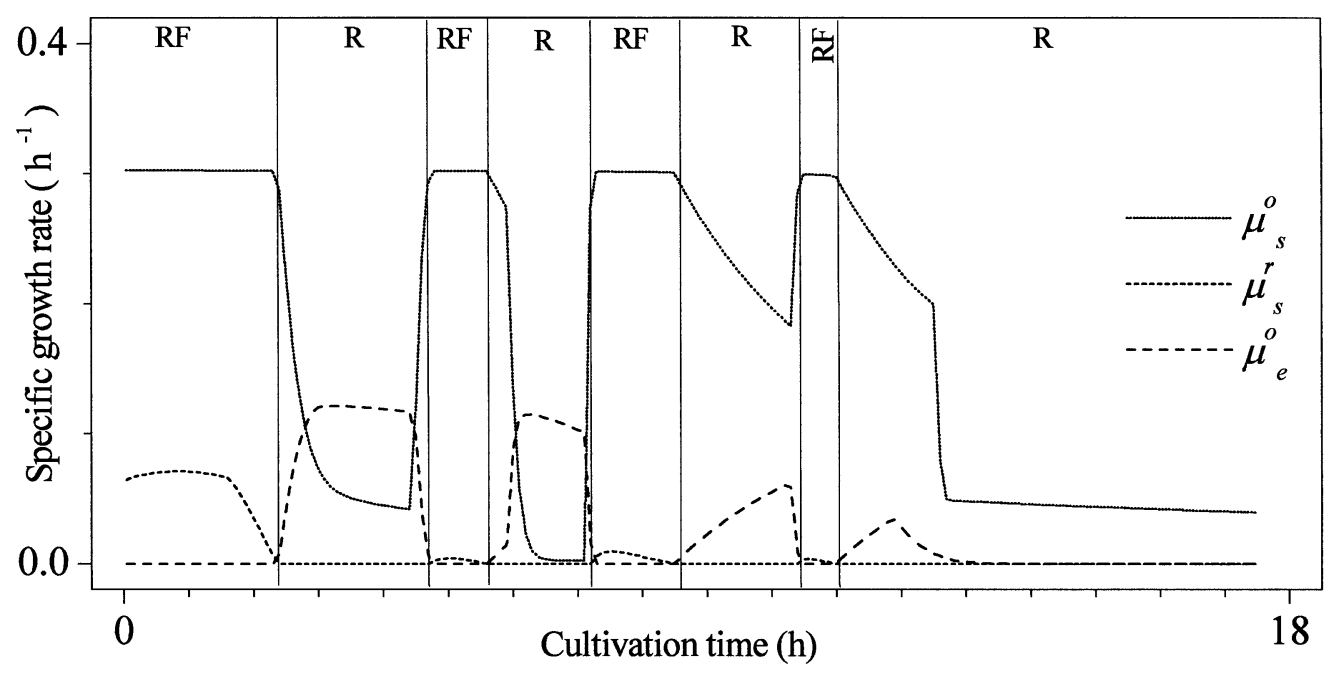

Fig. 2. "True" specific growth rate profiles for the case-study, employing the bottleneck kinetic model [18].

$$
\begin{aligned}
& \frac{\mathrm{d} \hat{\psi}}{\mathrm{d} t}= X\left[\begin{array}{l}
\hat{\mu}_{s}^{o} \\
\hat{\mu}_{s}^{r}
\end{array}\right]-D \psi+\left[\begin{array}{ll}
-k_{5} & 0 \\
k_{7} & k_{8}
\end{array}\right]^{-1}\left[\begin{array}{l}
\text { OTR } \\
-C T R
\end{array}\right] \\
&+\left[\begin{array}{ll}
\omega_{1} & 0 \\
0 & \omega_{2}
\end{array}\right](\psi-\hat{\psi}) \\
& \frac{\mathrm{d}}{\mathrm{d} t}\left(\left[\begin{array}{l}
\hat{\mu}_{s}^{o} \\
\hat{\mu}_{s}^{r}
\end{array}\right]\right)=X\left[\begin{array}{ll}
\gamma_{1} & 0 \\
0 & \gamma_{2}
\end{array}\right](\psi-\hat{\psi}),
\end{aligned}
$$

and for the respirative state:

$$
\begin{aligned}
& \psi= {\left[\begin{array}{cc}
-k_{5} & -k_{6} \\
k_{7} & k_{9}
\end{array}\right]^{-1}\left[\begin{array}{l}
C \\
G
\end{array}\right] } \\
& \frac{\mathrm{d} \hat{\psi}}{\mathrm{d} t}= X\left[\begin{array}{l}
\hat{\mu}_{s}^{o} \\
\hat{\mu}_{e}^{o}
\end{array}\right]-D \psi \\
&+\left[\begin{array}{cc}
-k_{5} & -k_{6} \\
k_{7} & k_{9}
\end{array}\right]^{-1}\left[\begin{array}{l}
O T R \\
-C T R
\end{array}\right] \\
&+\left[\begin{array}{cc}
w_{1} & 0 \\
0 & w_{3}
\end{array}\right](\psi-\hat{\psi}) \\
& \frac{\mathrm{d}}{\mathrm{d} t}\left(\left[\begin{array}{l}
\hat{\mu}_{s}^{o} \\
\hat{\mu}_{e}^{o}
\end{array}\right]\right)=X\left[\begin{array}{ll}
\gamma_{1} & 0 \\
0 & \gamma_{3}
\end{array}\right](\psi-\hat{\psi})
\end{aligned}
$$

In practical terms, variables $C, G, O T R, C T R$, and $F_{\text {in }}$ in Fig. $1 \mathrm{a}-\mathrm{c}$ are considered as process on-line measurements, being supplied to the estimation algorithms at a sampling period of 6 min. Also, it should be noted that Eqs. (46a) and (47a) correspond to the transformation defined by Eq. (7), but with two different $K_{a}^{-1}$ definitions, related to each of the two partial models employed.

Fig. 3 shows the results produced by the reduced-order OBE for three different tuning trials. The equations were integrated with a robust variable step integration algo-
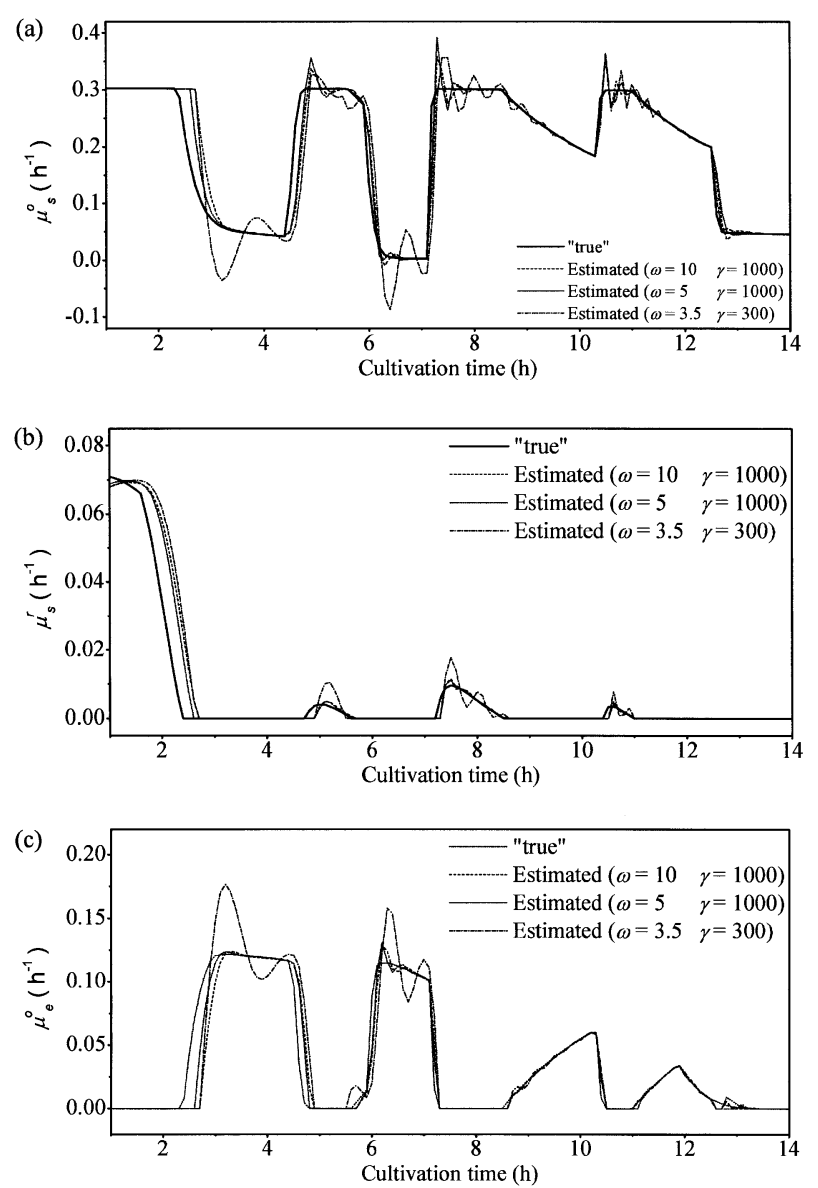

Fig. 3. Comparison between specific growth rate estimates given by the continuous reduced-order OBE (dotted lines) and the "true" values (full line) for different tuning parameters. (a) Respiratory glucose uptake pathway. (b) Fermentative glucose uptake pathway. (c) Respiratory ethanol uptake pathway.

rithm (4th/5th order Runge-Kutta type embedded scheme due to Butcher) employing along the integration linear estimates of the relevant sampled variables. 
The accuracy of the estimates can be assessed by the ITAE error index (ITAE: integral of time-weighted absolute errors) given in Table 2. On the third trial with $\omega_{1}=\omega_{2}=\omega_{3}=10$ and $\gamma_{1}=\gamma_{2}=\gamma_{3}=1000$ the results are quite satisfactory. However, a qualitative analysis of the plots shows that for all trials the dynamics of convergence is time-varying, i.e. the response becomes increasingly faster and oscillatory as the run approaches the end. This is typical of the OBE estimator. Stability is ensured but there is little control on the dynamics of convergence from estimated rates to 'true' rates.

\subsection{Tuning the $O B E$ to yield second order error dynamics}

The same principles discussed in the previous section were applied for designing the OBE with second order dynamics tuning [SODE - using estimator Eqs. (11) with tuning Eqs. (36) and (37)].

The resulting equations for the respiro-fermentative state are:

$$
\begin{aligned}
\psi= & {\left[\begin{array}{ll}
-k_{5} & 0 \\
k_{7} & k_{8}
\end{array}\right]^{-1}\left[\begin{array}{l}
C \\
G
\end{array}\right] } \\
\frac{\mathrm{d} \hat{\psi}}{\mathrm{d} t}= & X\left[\begin{array}{l}
\hat{\mu}_{s}^{o} \\
\hat{\mu}_{s}^{r}
\end{array}\right]-D \psi+\left[\begin{array}{ll}
-k_{5} & 0 \\
k_{7} & k_{8}
\end{array}\right]^{-1}\left[\begin{array}{l}
\text { OTR } \\
-C T R
\end{array}\right] \\
& +\left[\begin{array}{ll}
2 \zeta_{1} / \tau_{1}-\hat{\phi} & 0 \\
0 & 2 \zeta_{2} / \tau_{2}-\hat{\phi}
\end{array}\right](\psi-\hat{\psi})
\end{aligned}
$$

$$
\frac{\mathrm{d}}{\mathrm{d} t}\left(\left[\begin{array}{l}
\hat{\mu}_{s}^{o} \\
\hat{\mu}_{s}^{r}
\end{array}\right]\right)=\left[\begin{array}{ll}
\left(\tau_{1}^{2} X\right)^{-1} & 0 \\
0 & \left(\tau_{2}^{2} X\right)^{-1}
\end{array}\right](\psi-\hat{\psi}),
$$

and for the respirative state:

$$
\begin{aligned}
& \psi=\left[\begin{array}{cc}
-k_{5} & -k_{6} \\
k_{7} & k_{9}
\end{array}\right]^{-1}\left[\begin{array}{l}
C \\
G
\end{array}\right] \\
& \frac{\mathrm{d} \hat{\psi}}{\mathrm{d} t}=X\left[\begin{array}{l}
\hat{\mu}_{s}^{o} \\
\hat{\mu}_{e}^{o}
\end{array}\right]-D \psi+\left[\begin{array}{cc}
-k_{5} & -k_{6} \\
k_{7} & k_{9}
\end{array}\right]^{-1}\left[\begin{array}{l}
\text { OTR } \\
-C T R
\end{array}\right] \\
& +\left[\begin{array}{ll}
2 \zeta_{1} / \tau_{1}-\hat{\phi} & 0 \\
0 & 2 \zeta_{3} / \tau_{3}-\hat{\phi}
\end{array}\right](\psi-\hat{\psi})
\end{aligned}
$$

Table 2

ITAE for 3 different reduced-order OBE tuning sets

\begin{tabular}{llll}
\hline & \multicolumn{2}{l}{ ITAE } & \\
\cline { 2 - 4 } Tuning case & $\mu_{s}^{o}$ & $\mu_{s}^{r}$ & $\mu_{e}^{o}$ \\
\hline Case 1 $\left(\omega_{i}=3.5 \gamma_{i}=300\right)$ & 1.90 & 0.11 & 0.63 \\
Case 2 $\left(\omega_{i}=5 \gamma_{i}=1000\right)$ & 1.30 & 0.05 & 0.27 \\
Case 3 $\left(\omega_{i}=10 \gamma_{i}=1000\right)$ & 0.97 & 0.06 & 0.26 \\
\hline
\end{tabular}

$\frac{\mathrm{d}}{\mathrm{d} t}\left(\left[\begin{array}{l}\hat{\mu}_{s}^{o} \\ \hat{\mu}_{e}^{o}\end{array}\right]\right)=\left[\begin{array}{ll}\left(\tau_{1}^{2} X\right)^{-1} & 0 \\ 0 & \left(\tau_{3}^{2} X\right)^{-1}\end{array}\right](\psi-\hat{\psi})$

with

$\phi(X)=\frac{\ln \left(X_{t+1}\right)-\ln \left(X_{t}\right)}{T}$.

The results obtained with the SODE algorithm are depicted in Figs. $4 \mathrm{a}-\mathrm{c}$ and $5 \mathrm{a}-\mathrm{c}$. The equations were integrated with the same variable step integration algorithm employed for the reduced-order OBE. The influence of $\zeta$ can be assessed from the plots in Fig. $4 \mathrm{a}-\mathrm{c}$ where $\tau$ is kept constant for $0.15 \mathrm{~h}$ while $\zeta$ takes the values $0.25,0.5,1.0$ and 1.25 respectively. The ITAE error indexes for the 4 different tunings are given in Table 3. It is relevant to note that best results are obtained when underdamped trajectories are forced.
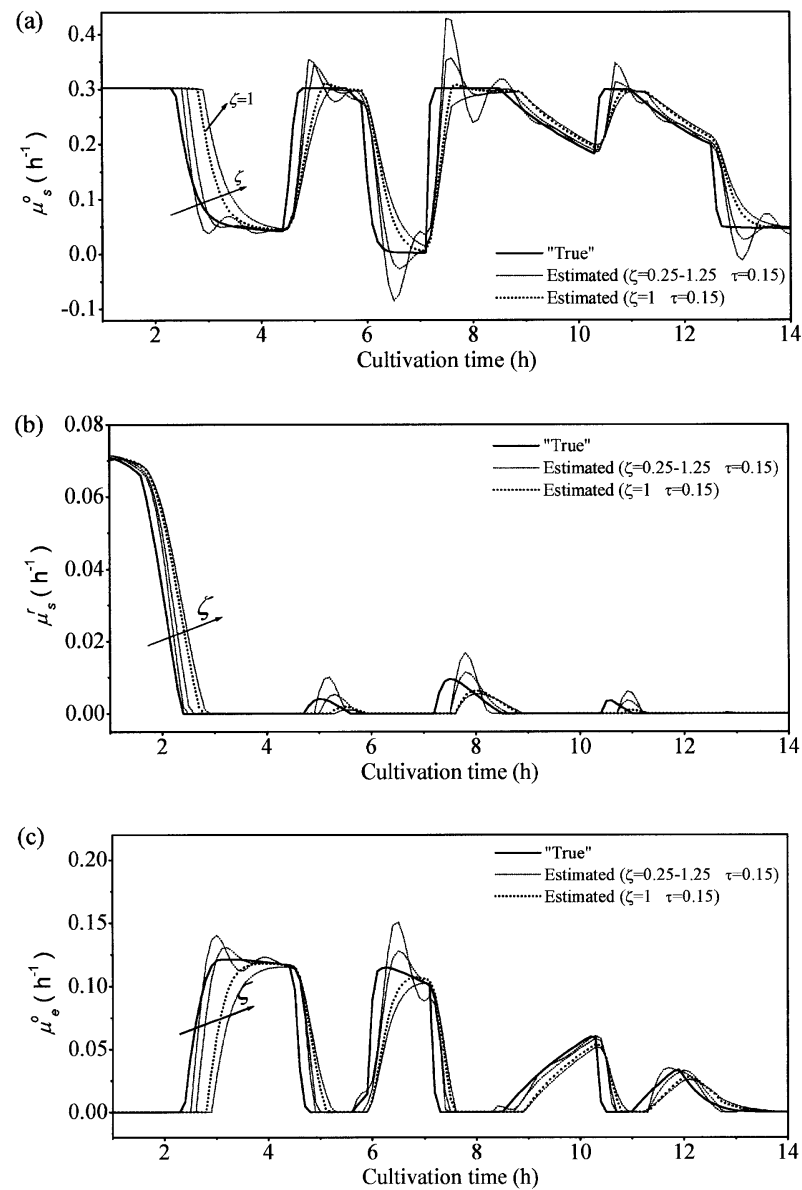

Fig. 4. Illustration of the influence of the damping coefficient $\zeta$ on the performance of the continuous SODE algorithm (a) respiratory glucose uptake pathway. (b) fermentative glucose uptake pathway. (c) Respiratory ethanol uptake pathway (specific growth rates estimatesdotted lines; "true values"-full lines). 
Table 3

ITAE for 4 different SODE tuning sets reflecting the influence of $\zeta$

\begin{tabular}{llll}
\hline & \multicolumn{2}{l}{ ITAE } & \\
\cline { 2 - 4 } Tuning case & $\mu_{s}^{o}$ & $\mu_{s}^{r}$ & $\mu_{e}^{o}$ \\
\hline Case 1 $\left(\tau_{i}=0.15 \zeta_{i}=0.25\right)$ & 2.90 & 0.10 & 0.73 \\
Case 2 $\left(\tau_{i}=0.15 \zeta_{i}=0.5\right)$ & 2.30 & 0.095 & 0.78 \\
Case 3 $\left(\tau_{i}=0.15 \zeta_{i}=1\right)$ & 3.2 & 0.11 & 1.2 \\
Case 4 $\left(\tau_{i}=0.15 \zeta_{i}=1.25\right)$ & 3.9 & 0.12 & 1.4 \\
\hline
\end{tabular}

The influence of $\tau$ can be assessed from the plots in Fig. 5a-c where $\zeta$ is kept constant for $1.0 \mathrm{~h}$ while $\tau$ takes the values $0.15,0.1,0.05$ and $0.01 \mathrm{~h}$ respectively. The ITAE error indexes for the 4 different tunings are given in Table 4.

As given by the plots in Figs. $4 \mathrm{a}-\mathrm{c}$ and $5 \mathrm{a}-\mathrm{c}$ the characteristics of the dynamics of convergence of the estimated values to the true values appears to be in agreement with typical second-order dynamical responses. It is shown that decreasing $\tau$ faster responses are
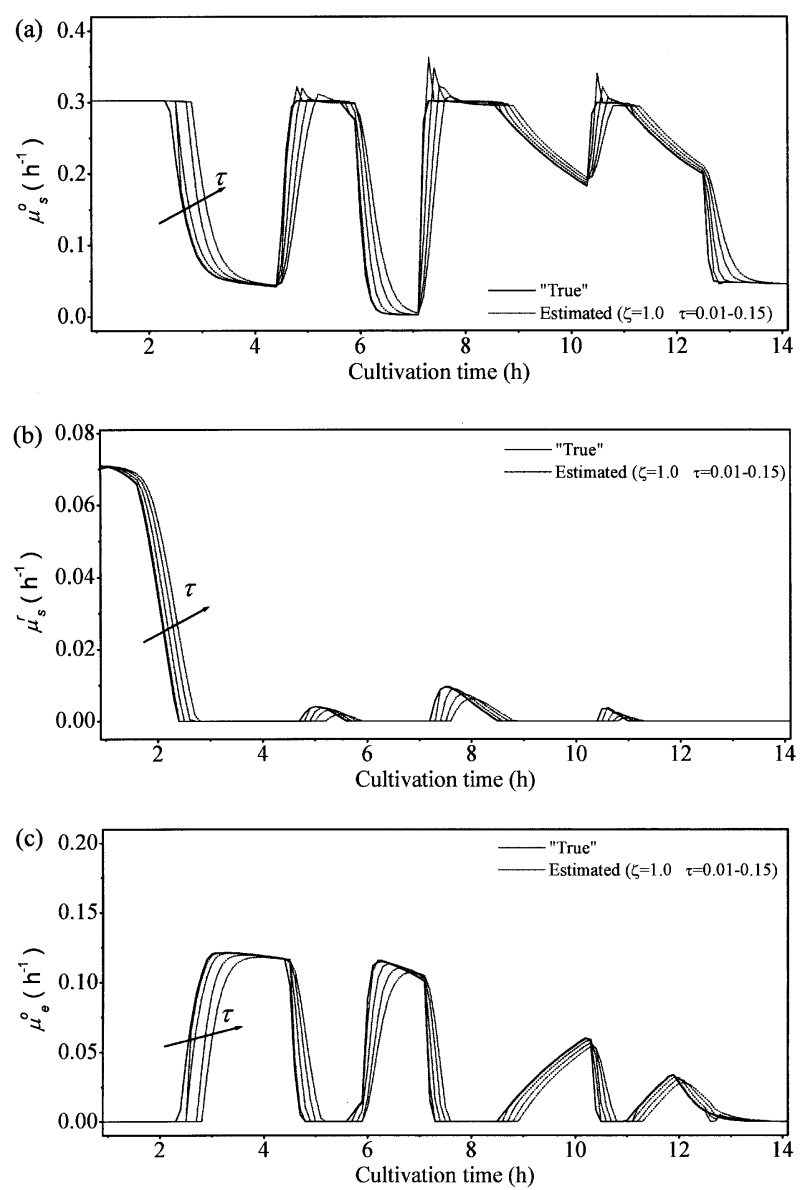

Fig. 5. Illustration of the influence of the natural period of oscillation $\tau$ on the performance of the continuous SODE algorithm: (a) respiratory glucose uptake pathway. (b) Fermentative glucose uptake pathway. (c) Respiratory ethanol uptake pathway. (specific growth rates estimates - dotted lines; "true values"-full lines).
Table 4

ITAE for 4 different SODE tuning sets reflecting the influence of $\tau$

\begin{tabular}{llll}
\hline & \multicolumn{3}{l}{ ITAE } \\
\cline { 2 - 4 } Tuning case & $\mu_{s}^{o}$ & $\mu_{s}^{r}$ & $\mu_{e}^{o}$ \\
\hline Case 1 $\left(\tau_{i}=0.01 \zeta_{i}=1\right)$ & 0.43 & 0.015 & 0.14 \\
Case 2 $\left(\tau_{i}=0.05 \zeta_{i}=1\right)$ & 1.2 & 0.051 & 0.42 \\
Case 3 $\left(\tau_{i}=0.1 \zeta_{i}=1\right)$ & 2.2 & 0.086 & 0.83 \\
Case 4 $\left(\tau_{i}=0.15 \zeta_{I}=1\right)$ & 3.2 & 0.110 & 1.20 \\
\hline
\end{tabular}

produced and estimation errors are decreased (Table 4). Decreasing $\zeta$ produces more oscillatory responses. Furthermore, from the plots in Fig. $4 \mathrm{a}-\mathrm{c}$ it can be concluded that $\zeta=1$ constitutes the frontier between oscillatory and non-oscillatory behaviour (as expected). These results illustrate the advantage of employing a formulation where the tuning parameters have a well established meaning thus simplifying the search for optimum tuning parameters.

\subsection{Discrete-time SODE implementation}

In Section 3.3 the numerical implementation of the SODE algorithm and its relation with stability constraints was studied. It was shown that a forward Euler discretisation of the continuous equations poses stability problems. In particular, three relations were derived which define stable intervals for the integration step $T$ in relation to specific tuning sets (Table 1). This is an interesting and relevant practical issue, illustrated next in Figs. 6 and 7.

In industrial (or pilot, or laboratory) applications, sampling periods are generally set based on considerations external to this convergence problem, this meaning that when tuning the estimator the sampling period parameter is already fixed. The consequence is that an effective constraint exists, and must be obeyed, between the damping coefficient and the natural period of oscillation.

Figs. 6 and 7 aim thus at illustrating the behaviour of the SODE with an Euler discretisation, when the stability

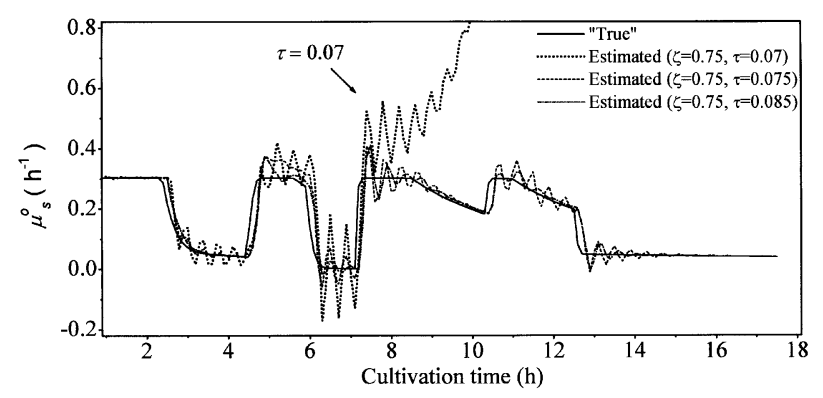

Fig. 6. Illustration of stability constraints in the application of the discrete-time SODE on the estimation of specific growth rates (estimates - dotted lines; "true" values - full lines) for case-studies with $\zeta=0.75(\tau=0.07, \tau=0.075$ and $\tau=0.085)$. 


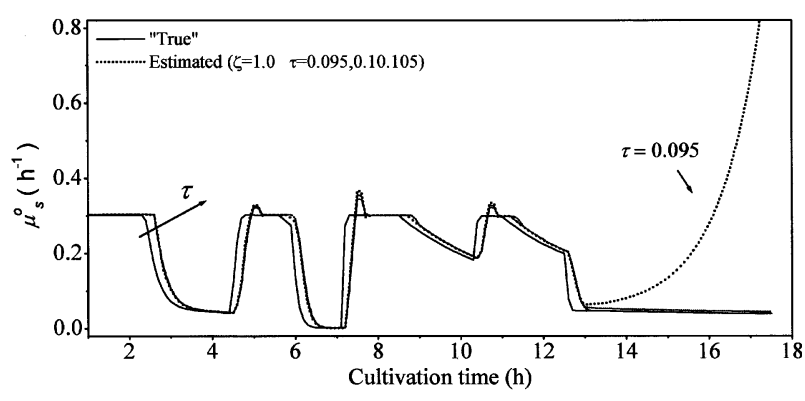

Fig. 7. Illustration of stability constraints in the application of the discrete-time SODE on the estimation of specific growth rates (estimates - dotted lines; "true" values - full lines) for case-studies with $\zeta=01.0(\tau=0.095, \tau=0.1$ and $\tau=0.105)$.

limits are violated. The integration step and the sampling time were assumed to be $6 \mathrm{~min}$.

The results in Fig. 6 were obtained with $\zeta=0.75$ for three alternative values $\tau=0.07, \tau=0.075$ and $\tau=0.085$ hours. As given in Table 1, if $T=0.1 \mathrm{~h}$ then $\tau$ must be larger than 0.07 for stability. This is confirmed by the diverging trajectory depicted in the figure for $\tau=0.07$. Increasing $\tau$ to 0.075 divergence is no more observed. Nevertheless, persistent oscillations are exhibited suggesting that the estimator operates near the stability limit. For $\tau=0.085$ a normal stable output is obtained.

The results in Fig. 7 were obtained with $\zeta=1.0$ for $\tau=0.095, \tau=0.1$ and $\tau=0.105$. As given in Table 1 , if $T=0.1 \mathrm{~h}$ and $\zeta=1.0$ then $\tau$ must be larger than 0.1 . The plotting of results again confirm this limit for stability: with $\tau=0.095$ the algorithm leads to divergence; increasing to 0.1 divergence is no more observed, but the response exhibits some overshoot which is not characteristic of a second-order response with $\zeta=1.0$, suggesting that the estimator operates near the stability limit; for $\tau=0.105$ the overshoot is eliminated as expected of an algorithm operating within stable regions.

\section{Conclusions}

The application of model-based algorithms for bioprocess operation is often hindered by the complexity of micro-organism growth kinetics and the related limited knowledge of critical bioprocess parameters. Several algorithms are available today that, with some limited requirements fulfilled, allow both the on-line estimation of key bioprocess variables and the on-line identification of relevant kinetic characteristic parameters. The tuning of such algorithms is in many cases not a trivial procedure, related as it is (or as it should be) to stability and tracking properties. This is an important issue that may represent a source of problems limiting the acceptance of such algorithms for practical use in industry.

In the present work we characterised in detail the relationships between tuning, stability and dynamics of convergence in observer-based kinetics estimators for stirred-tank bioreactors, both for continuous and for discrete time formulations of the estimation algorithms.

For the former cases it was shown that with proper tuning the user may guarantee stability and simultaneously impose a desired quasi-time invariant second order time response (underdamped, critically damped or overdamped) for the convergence of estimates to true values. In practical terms this tuning is just the setting of the parameters characteristic of second-order responses, the damping coefficient and the natural period of oscillation.

For the latter case, an algorithm was developed based on a forward Euler discretisation whose discrete time error system is shown to be linear time-invariant. Furthermore, stability conditions were derived, which define the stable domain for the discretisation (sampling) period as function of the second order tuning parameters.

The theory presented was illustrated with a case-study of Baker's yeast fermentation, employing as source of data a process model validated by laboratory experiments. The results for both approaches very clearly confirmed the theoretical developments. In particular, the results concerning the stability domain for the Euler-based discrete time formulation of the estimator were shown to have important practical implications.

With the present work we expect to contribute for the understanding of the scope of this class of algorithms and through the simplification of the tuning procedures to improve their acceptance for practical applications.

\section{References}

[1] A. Munack, An optimal feeding strategy for identification of Monod-type models by fed batch experiments, in: N.M. Fish, R.I. Fox, N.F. Thornhill (Eds.), Computer Applications in Fermentation Technology: Modelling and Control of Biotechnological Processes, Elsevier Applied Science, London, 1989, pp. 195-204.

[2] M. Baltes, R. Schneider, C. Sturm, M. Reuss, Optimal experimental design for parameter estimation in unstructured growth models, Biotechnol. Prog. 10 (1994) 480-488.

[3] C. Rocha, E.C. Ferreira, Design of optimal experiments for identification of yield coefficients in a Baker's yeast model, in: B. Glennon, P.M. Kieran, K.Ch.A.M. Luyben (Eds.), 1st European Symposium on Biochemical Engineering Science, Dublin 1996, pp. 99-100.

[4] G. Bastin, D. Dochain, On-Line Estimation and Adaptive Control of Bioreactors, Elsevier, Amsterdam, 1990.

[5] M. Farza, S. Othman, H. Hammouri, J. Biston, A nonlinear approach for the on-line estimation of the kinetic rates in bioreactors. Application to a lactic acid production process, Bioprocess Eng. 17 (1997) 143-150.

[6] M. Farza, H. Hammouri, S. Othman, K. Busawon, Nonlinear observers for parameter estimation in bioprocesses, Chemical Engineering Science 52 (23) (1997) 4251-4267.

[7] M. Farza, K. Busawon, H. Hammouri, Simple nonlinear observers for on-line estimation of kinetic rates in bioreactors, Automatica 34 (3) (1998) 301-318.

[8] M. Farza, H. Hammouri, C. Jallut, J. Lieto, State observation of a nonlinear system: application to (bio)chemical processes, AIChE J.ournal 45 (1) (1999) 93-106. 
[9] M. Farza, S. Othman, H. Hammouri, M. Fick, Discrete-time nonlinear observer-based estimators for the on-line estimation of the kinetic rates in bioreactors, Bioprocess Eng. 17 (1997) 247-255.

[10] M.U. Estler, Recursive on-line estimation of the specific growth rate from off-gas analysis for the adaptive control of fed-batch processes, Bioprocess Eng. 12 (1995) 205-207.

[11] V.N. Lubenova, Stable adaptive algorithm for simultaneous estimation of time-varying parameters and state variables in aerobic bioprocesses, Bioprocess Eng. 21 (3) (1999) 219-226.

[12] M. Perrier, S. Feyo de Azevedo, E.C. Ferreira, D. Dochain, Tuning of observer-based estimators: theory and application to the on-line estimation of kinetic parameters, Control Engineering Practice 8 (4) (2000) 377-388.

[13] D. Dochain, M. Perrier, B.E. Ydstie, Asymptotic observers for stirred tank reactors, Chem. Eng. Sci. 47 (1991) 4167-4178.

[14] Y. Pomerleau, M. Perrier, Estimation of multiple specific growth rates in bioprocesses, AIChE J.ournal 36 (2) (1990) 207-215.
[15] R. Oliveira, E.C. Ferreira, F. Oliveira, S. Feyo de Azevedo, A study on the convergence of observer-based kinetic estimators in fed-batch fermentations, J. Process Control 6 (6) (1996) 367371.

[16] K.S. Narendra, A.M. Annaswany, Stable Adaptive Systems, Prentice Hall, Englewood Cliffs, 1989.

[17] S. Feyo de Azevedo, P. Pimenta, E.C. Ferreira, F. Oliveira, Studies on on-line state and parameter estimation through a realtime process simulator, in: G. Stephanopoulos, N. Karim (Eds.), Modeling and Control of Biotechnical Processes (2nd IFAC Symposium) and Computer Applications to Fermentation Technology (5th International Conference), Pergamon Press, Oxford 1992, 1992, pp. 453-458.

[18] B. Sonnleitner, O. Käppeli, Growth of Saccharomyces cerevisiae is controlled by its limited respiratory capacity: formulation and verification of a hypothesis, Biotech. Bioeng. 28 (1986) 927-937. 